Revue d'histoire de l'Amérique française

Q4 REVUE D.HISTOIRE DE L'AMÉRIQUE FRANÇAISE

\title{
Mouvements migratoires et familles : le peuplement du Saguenay avant 1911
}

\section{Danielle Gauvreau et Mario Bourque}

Volume 42, numéro 2, automne 1988

URI : https://id.erudit.org/iderudit/304677ar

DOI : https://doi.org/10.7202/304677ar

Aller au sommaire du numéro

\section{Éditeur(s)}

Institut d'histoire de l'Amérique française

\section{ISSN}

0035-2357 (imprimé)

1492-1383 (numérique)

Découvrir la revue

\section{Citer cet article}

Gauvreau, D. \& Bourque, M. (1988). Mouvements migratoires et familles : le peuplement du Saguenay avant 1911. Revue d'histoire de l'Amérique française, 42(2), 167-192. https://doi.org/10.7202/304677ar
Résumé de l'article

L'histoire de la société québécoise est marquée dans la seconde moitié du $19^{\mathrm{e}}$ siècle par l'importance des mouvements migratoires. En dépit d'une situation économique et sociale en mutation, ces mouvements ne paraissent pas s'inscrire en rupture complète avec le modèle de reproduction familiale qui s'appuie déjà largement sur la mobilité géographique.

Près de 30000 personnes ont alimenté le mouvement de colonisation du Saguenay de 1838 à 1911, ce qui paraît bien peu comparativement par exemple au nombre de personnes qui ont émigré aux États-Unis à la même époque. Dès 1870 d'ailleurs, la balance migratoire régionale est négative et la population doit compter sur sa propre vigueur démographique pour se reproduire. L'analyse démontre bien le caractère familial de l'immigration, qui touche surtout des couples et des familles. Avec l'importance initiale du courant Charlevoix-Saguenay, ce trait paraît à l'origine de l'homogénéité de la population saguenayenne, particulièrement du point de vue génétique.
Tous droits réservés @ Institut d'histoire de l'Amérique française, 1988
Ce document est protégé par la loi sur le droit d'auteur. L'utilisation des services d'Érudit (y compris la reproduction) est assujettie à sa politique d'utilisation que vous pouvez consulter en ligne.

https://apropos.erudit.org/fr/usagers/politique-dutilisation/ 


\title{
MOUVEMENTS MIGRATOIRES ET FAMILLES: LE PEUPLEMENT DU SAGUENAY AVANT 1911 ${ }^{1}$
}

\author{
DANIELLE GAUVREAU \\ MARIO BOURQUE \\ SOREP \\ Université du Québec à Chicoutimi
}

\section{RÉSUMÉ}

L'histoire de la société québécoise est marquée dans la seconde moitié du 19e siècle par l'importance des mouvements migratoires. En dépit d'une situation économique et sociale en mutation, ces mouvements ne paraissent pas s'inscrire en rupture complète avec le modèle de reproduction familiale qui s'appuie déjà largement sur la mobilité géographique.

Près de 30000 personnes ont alimenté le mouvement de colonisation du Saguenay de 1838 à 1911, ce qui paraît bien peu comparativement par exemple au nombre de personnes qui ont émigré aux Etats-Unis à la même époque. Dès 1870 d'ailleurs, la balance migratoire régionale est négative et la population doit compter sur sa propre vigueur démographique pour se reproduire. L'analyse démontre bien le caractère familial de l'immigration, qui touche surtout des couples et des familles. Avec l'importance initiale du courant Charlevoix-Saguenay, ce trait paraît à l'origine de l'homogénéité de la population saguenayenne, particulièrement du point de vue génétique.

\section{ABSTRACT}

Geographic mobility was important in Québec during the second half of the nineteenth century. This situation was not completely new, as migration had always been an important demographic component; but it surely became more common at that time and could have been directed to new regions that were opening following recent economic changes.

A total of 30000 persons arrived in Saguenay between 1838 and 1911; it is a small number compared to the number of those who left the Québec province for the United States during the same period. The analysis shows that the Saguenay migratory balance was negative as soon as 1870: the growth of the population was then only due to natural increase. Most immigrants came within couples or families, a result which illustrates the familial character of migration. This seems to be responsible for the homogeneity of the Saguenay population, particularly evident at the genetic level. This homogeneity is also due to the strength of the migratory stream established at the beginning between Charlevoix and Saguenay.

1 Cet article consiste en une version remaniée d'une communication qui a été présentée au congrès de la Société historique du Canada en juin 1987 à Hamilton. Nous voulons remercier tous les chercheurs de SOREP qui ont contribué à ce travail ou l'ont commenté à quelque moment que ce soit. Cette recherche a par ailleurs été subventionnée par le Conseil de recherches en sciences humaines du Canada (CRSHC) et le Fonds pour la formation de chercheurs et l'aide à la recherche du Québec (FCAR). 


\section{1 - STRATÉGIES MIGRATOIRES ET REPRODUCTION FAMILIALE}

L'histoire de la population québécoise est marquée par l'importance des mouvements migratoires, tant internationaux qu'internes. Le peuplement blanc résulte d'abord au 17e siècle d'un faible mouvement de colonisation en provenance de la France ${ }^{2}$. Le peuplement progressif du territoire québécois s'effectue quant à lui sous la poussée de déplacements internes dont tout indique qu'ils s'inscrivent dans un régime démographique propre aux sociétés coloniales pré-industrielles. Le modèle de reproduction familiale proposé par Gérard Bouchard pour les systèmes ouverts ${ }^{3}$ paraît s'appliquer ici: dans un pays neuf où la terre est disponible, la migration constitue une stratégie familiale à laquelle on a recours pour pourvoir à l'établissement des enfants, lorsque celui-ci ne peut être assuré dans la communauté d'origine. Ainsi, la reproduction familiale s'effectue sur place par la transmission et la consolidation des terres d'une génération à l'autre (le modèle le plus connu), mais elle s'effectue aussi par la colonisation et le développement de nouvelles aires de peuplement ${ }^{4}$. Malgré l'absence quasi totale d'études menées à partir du lieu d'origine, on peut penser que la migration ne touche pas toutes les familles de la même façon, que ce soit en raison d'un avantage économique ${ }^{5}$ ou d'une charge démographique moins lourde. Les stratégies élaborées peuvent également varier: toute la famille peut migrer, laissant derrière elle des terres que d'autres pourront acquérir pour combler leurs propres besoins; une partie seulement de la famille peut aussi faire souche dans un nouvel endroit.

Le Québec connaît au cours de la seconde moitié du 19e siècle des mouvements migratoires d'une rare intensité: colonisation des régions éloignées des centres de la vallée du Saint-Laurent, émigration vers

\footnotetext{
2 Sur cette question, voir Hubert Charbonneau, Bertrand Desjardins, André Guillemette, Yves Landry, Jacques Légaré et François Nault, Naissance d' une population. Les Français établis au Canada au XVIIe siècle (Paris/Montréal, Institut national d'études démographiques, Presses universitaires de France/Presses de l'Université de Montréal, coll. «Travaux et documents», no $118,1987)$ chapitre 1.

3 Voir la description qu'en fait Gérard Bouchard pour le Saguenay dans «Sur la reproduction en milieu rural: systèmes ouverts et systèmes clos», Recherches sociographiques, 28,2-3 (1987): 229-252. L'idée de base était déjà formulée dans «Démographie et société rurale au Saguenay, 1851-1935», Recherches sociographiques, 19,1 (1978): 7-31 (du même auteur). Les autres éléments qui caractérisent ce régime démographique sont vraisemblablement une nuptialité précoce et des niveaux de fécondité élevés, réduits cependant par des niveaux de mortalité également importants.

4 En Nouvelle-France, ce modèle paraît confirmé par quelques travaux récents portant sur la composition du mouvement migratoire à l'origine de nouvelles paroisses. Voir Yves Beauregard et al., "Famille, parenté et colonisation en Nouvelle-France», Revue d'histoire de l'Amérique française, 39,3 (hiver 1986): 391-405, et Jacques Mathieu, «Mobilité et sédentarité: stratégies familiales en Nouvelle-France», Recherches sociographiques, 28,2-3 (1987): 211-227.

5 Sur les inégalités dans la société rurale, voir notamment Christian Dessureault, «L'égalitarisme paysan dans l'ancienne société rurale de la vallée du Saint-Laurent: éléments pour une réinterprétation», Revue d' histoire de l'Amérique française, 40,3 (hiver 1987): 373-407.
} 
l'Ouest canadien, migrations internes vers les centres urbains qui commencent à s'industrialiser ${ }^{6}$ et, surtout, émigration vers les ÉtatsUnis, en direction en particulier des usines de textile de la NouvelleAngleterre $^{7}$. Ce dernier courant atteint ses niveaux les plus élevés à la fin du 19e siècle: au total, on estime à au moins 500000 le nombre de Québécois et Québécoises ayant émigré vers les États-Unis entre 1850 et $1900^{8}$. Rappelons que durant la même période, le Québec reçoit de l'extérieur un nombre considérable d'immigrants et d'immigrantes à la quête d'un emploi dans la structure industrielle et urbaine naissante.

Le moteur de ces mouvements est largement économique et il s'inscrit dans la logique du développement capitaliste en cours à cette époque. Ainsi en est-il des usines de textile de la Nouvelle-Angleterre ou du développement industriel de Montréal. Le mouvement de colonisation lui-même n'échappe pas totalement à cette logique, avec la présence des compagnies forestières qui vont puiser dans les nouvelles régions des matières premières d'une importance stratégique. Toutefois, cet appel largement économique à émigrer vers la NouvelleAngleterre, Montréal ou une région de colonisation récente ne peut être entendu que parce qu'il tombe en terrain propice. D'une part, il s'accorde avec la logique du régime démographique qui fait déjà une large place à la mobilité géographique et, d'autre part, il s'inscrit dans une conjoncture particulière pour l'agriculture québécoise. Plusieurs interprétations ont été proposées pour expliquer l'évolution du Québec rural au cours du $19 \mathrm{e}$ siècle ${ }^{9}$. Serge Courville a récemment souligné la difficulté de faire la lumière sur cette question et suggéré une interprétation différenciée: il soutient qu'en certains endroits, l'agriculture s'articule de plus en plus aux marchés en même temps qu'apparaissent des industries rurales, «créant un contexte nouveau de développement, signalant sans doute moins «une crise agricole» que l'une des premières crises de l'âge industriel ${ }^{10}$. Ailleurs le long de l'axe laurentien (par exemple dans Charlevoix), le problème se pose plutôt en termes de saturation des terres. Les limites de l'espace laurentien se trouvent bientôt atteintes,

6 La proportion de la population urbaine augmente au Québec dès le milieu du $19 \mathrm{e}$ siècle, passant de $14,9 \%$ en 1851 à $23,8 \%$ en 1881 . Leroy Stone, Urban Development in Canada, cité dans Paul-André Linteau, René Durocher et Jean-Claude Robert, Histoire du Québec contemporain, 1: De la confédération à la crise (1867-1929) (Montréal, Boréal Express, 1979), 151.

7 Le reste du Canada connaît lui aussi un exode massif vers les États-Unis. Voir Yolande Lavoie, L'émigration des Canadiens aux États-Unis avant 1930 (Montréal, Les Presses de l'Université de Montréal, 1972), 87 p.

8 Yolande Lavoie, «Les mouvements migratoires des Canadiens entre leur pays et les ÉtatsUnis au XIXe et au XXe siècles: étude quantitative», Hubert Charbonneau, éd., La population du Québec: études rétrospectives (Montréal, Boréal Express, 1973), 78.

9 Voir, en particulier, les nombreuses études publiées par Fernand Ouellet et par Jean-Pierre Wallot et Gilles Paquet.

10 Serge Courville, «L'habitant canadien dans la première moitié du XIXe siècle: survie ou survivance?», Recherches sociographiques, 27,2 (1986): 193. 
forçant les migrations vers des terres plus reculées et moins propres à la culture, tandis que les vieilles propriétés sont de plus en plus morcelées par les pratiques de partage ${ }^{11}$.

Ces nouvelles situations modifient de diverses façons le régime démographique antérieur. Le changement le plus profond se fait vraisemblablement dans les campagnes qui commencent à s'industrialiser et là où l'économie de marché structure de plus en plus l'agriculture. C'est du moins ce que suggèrent les travaux d'Allan Greer sur trois paroisses du Bas-Richelieu entre 1740 et $1840^{12}$. Ainsi, à Sorel, la diminution de l'âge au mariage des hommes, un redressement de la balance migratoire et une croissance rapide de la population accompagnent l'industrialisation de la paroisse. Ailleurs, le changement apparaît davantage dans l'éventail de choix qui s'offrent désormais aux familles dont l'établissement des enfants n'est pas assuré sur place.

Le calendrier du mouvement de colonisation devance probablement celui des deux autres mouvements, mais seulement pour un temps, comme en témoigne la véritable saignée qui s'effectue en direction des États-Unis à partir surtout des années 1870. Comme Bruno Ramirez le suggère, la popularité de ce dernier choix tient probablement au caractère plus immédiat des avantages monétaires qui en découlent, en même temps qu'à la variété des stratégies qu'elle permet: migration temporaire ou permanente, migration de toute la famille ou de certains de ses membres qui restent intégrés au groupe familial ${ }^{13}$. Ce choix apparaît d'autant plus logique qu'il permet de compter sur les revenus de plusieurs membres de la famille et qu'il s'effectue dans un contexte culturel et social qui n'entraîne pas le déracinement total ${ }^{14}$.

Peu importe la destination retenue cependant, la façon de résoudre le dilemme initial est marquée par la continuité, du moins dans son caractère largement familial. En effet, diverses études portant sur des mouvements migratoires de la seconde moitié du $19 \mathrm{e}$ siècle attestent le caractère familial de l'émigration. Daniel Maisonneuve ${ }^{15}$ et France

\footnotetext{
11 Gérard Bouchard, «Sur l'historiographie des campagnes et des régions du Québec aux XIXe et XXe siècles: nouvelles propositions», Histoire sociale, sensibilités collectives et mentalités. Mélanges Robert Mandrou (Paris, Presses universitaires de France, 1985), 561-571.

12 Allan Greer, Peasant, Lord and Merchant. Rural Society in Three Quebec Parishes, 1740-1840 (Toronto, University of Toronto Press, 1985), 188-193.

13 Bruno Ramirez, The Crossroad Province: Québec's Place in International Migrations, 1870-1915. Communication présentée au symposium international «A Century of European Migrations 1830-1930 in Comparative Perspective», St.Paul, Minn., Novembre 1986.

14 Voir l'étude approfondie du cas de l'Amoskeag Company à Manchester par Tamara $\mathrm{K}$. Hareven, Family Time and Industrial Time (Cambridge, Cambridge University Press, 1982), $474 \mathrm{p}$.

is Daniel Maisonneuve, «Structure familiale et exode rural: le cas de Saint-Damase, 18521861», Cahiers québécois de démographie, 14,2 (1985): 231-240.
} 
Gagnon ${ }^{16}$ en font tous deux état à leur façon dans un contexte de peuplement ancien et d'agriculture prospère, le premier pour la paroisse de Saint-Damase, près de Saint-Hyacinthe, entre 1852 et 1861, et la seconde pour des paroisses de la rive nord de la plaine de Montréal entre 1845 et 1875. Les travaux de Tamara Hareven démontrent pour leur part la base familiale des réseaux migratoires et l'importance des liens familiaux dans l'émigration vers les villes de la Nouvelle-Angleterre ${ }^{17}$. Ces études s'inscrivent en outre dans un courant plus large qui a récemment démontré l'importance de la famille et des liens de parenté en général dans le phénomène migratoire ${ }^{18}$.

Dans la foulée de ces travaux, le présent article porte sur un volet spécifique des mouvements migratoires qui marquent le Québec à partir du milieu du 19e siècle: la colonisation du Saguenay/Lac-Saint-Jean (désormais le Saguenay). Il vise d'abord à mesurer l'ampleur du mouvement d'immigration qui a touché le Saguenay jusqu'au début du $20 \mathrm{e}$ siècle $^{19}$. Puis, partant de l'hypothèse que les migrations constituent une pièce maîtresse de la dynamique de reproduction familiale, il vise à caractériser ce mouvement de façon à mettre en évidence le profil démographique des personnes concernées ainsi que le caractère familial de l'immigration. Plus concrètement, il cherche donc à répondre aux questions suivantes: combien d'immigrants et d'immigrantes sont venus au Saguenay? Leur arrivée se faisait-elle en couples, en familles plus ou moins étendues, ou encore de façon isolée? D'où venaient ces personnes? Comment s'articule la dynamique migratoire à laquelle ils participaient à l'évolution démographique plus globale de la région et du Québec?

La banque de données constituée à Chicoutimi par SOREP sera utilisée pour répondre à ces questions. Elle renferme les familles reconstituées à partir des actes de baptême, mariage et sépulture contenus dans les registres paroissiaux saguenayens ${ }^{20}$. Soulignons qu'au-

\footnotetext{
16 France Gagnon, Le rôle de la famille dans l'adaptation des migrants de la plaine de Montréal au milieu montréalais, 1845-1875. Mémoire de maîtrise (histoire), Université du Québec à Montréal, 1986.

17 Tamara K. Hareven (1982), op. cit.

18 En ce qui concerne les migrations internationales, mentionnons Robert C. Ostergren, qui étudie l'émigration d'un village suédois vers le Mid-ouest américain, «Kinship Networds and Migration. A Nineteenth Century Swedish Example», Social Science History, 6,3 (1982): 293320; et Maureen Molloy, dont l'étude porte sur l'apparentement des immigrants des Highlands en Écosse vers le Cap-Breton, puis vers la Nouvelle-Zélande, «'No Inclination to Mix With Strangers': Marriage Patterns Among Highland Scots Migrants to Cape Breton and New Zealand (18001916)», Journal of Family History, 11,3 (1986): 221-243. Le peuplement initial du Canada ne suit toutefois qu'exceptionnellement ce modèle. Voir Charbonneau et al. (1987), op . cit., chapitre 3 .

19 C'est-à-dire à peu près jusqu'au moment de l'industrialisation de la région. Ce découpage est également dicté par les sources: au début de notre enquête en 1985, la banque de SOREP ne pouvait encore être utilisée au-delà de 1911 pour ce genre d'analyses.

20 Gérard Bouchard, Raymond Roy et Bernard Casgrain, Reconstitution automatique des familles. Le système SOREP (Chicoutimi, Université du Québec à Chicoutimi, dossier no 2, 1985), 2 volumes.
} 
delà de sa dimension historique et démographique, la présente étude s'inscrit dans un projet à caractère génétique qui vise à connaître le mode de constitution du bassin génétique saguenayen, caractérisé aujourd'hui par la prévalence élevée de certaines maladies héréditaires ${ }^{21}$.

\section{2 - LE PEUPLEMENT DU SAGUENAY}

La région du Saguenay (carte 1) s'ouvre à la colonisation blanche vers le milieu du 19e siècle (1838-1840) en réponse aux pressions qu'exercent les agriculteurs des régions plus anciennes du Québec, de même qu'en raison de l'attrait exercé par les forêts du Saguenay auprès des industriels forestiers. Dans Charlevoix, en particulier, l'ouverture de nouvelles paroisses sur les plateaux de l'arrière-pays (Saint-Urbain en 1827, Sainte-Agnès en 1833) ne suffit pas à combler les besoins en provenance du littoral, occupé progressivement depuis la fin du $17 \mathrm{e}$ siècle $^{22}$. Aussi, la colonisation du Saguenay est-elle en fait déjà amorcée lorsque prend fin en 1842 le bail que détient la Compagnie de la Baie d'Hudson qui fait de la région un domaine réservé, et le mouvement connaît alors une accélération rapide ${ }^{23}$. Il remonte d'abord le cours du Saguenay, les colons s'installent le long de la rivière, comme en témoignent les premiers recensements. Le Lac-Saint-Jean ne tarde pas toutefois à s'ouvrir à son tour à la colonisation et compte même plus de la moitié de la population de la région au tournant du 20e siècle. En 1852 , la population du Saguenay compte un peu plus de 5000 personnes; en 1911, ce chiffre s'est multiplié par dix.

Si la croissance rapide de la population résulte surtout dans un premier temps de l'apport migratoire, il n'en va plus de même dès les années 1870 (tableau 1). En effet, les taux de migration nette présentent à partir de cette date des valeurs négatives, montrant par là que le nombre de sorties dépasse celui des entrées. Ainsi, le mouvement d'immigration qui donne le coup d'envoi à la région paraît compensé relativement tôt par un mouvement inverse d'émigration. Cette tendance est particulièrement marquée au cours de la décennie 1882-1891, qui coïncide pour le Québec en général avec une période de forte émigration vers les États-Unis ${ }^{24}$. Pendant toute la période antérieure à 1911, les taux

21 Voir Gérard Bouchard, Claude Laberge et Charles R. Scriver, «La tyrosinémie héréditaire et le rachitisme vitamino-dépendant au Saguenay. Une approche génétique et démographique», L'Union médicale du Canada, 114 (1985): 633-636. Sur l'étroitesse des liens que peuvent entretenir et qu'entretiennent l'histoire sociale et la génétique humaine, voir «Un nouveau territoire pour l'historien? Vers une rencontre de l'histoire sociale et de la génétique humaine», un entretien d'Hubert Watelet avec Gérard Bouchard, à paraître dans Histoire sociale.

22 Voir entre autres Raoul Blanchard, L'Est du Canada français, Province de Québec (Montréal, Beauchemin, 1935), volume 1.

23 Voir Gérard Bouchard, «Le peuplement blanc», Christian Pouyez, Yolande Lavoie et al., Les Saguenayens. Introduction à l'histoire des populations du Saguenay, 16e-20e siècles (Québec, Presses de l'Université du Québec, 1983), 510 p.

24 Yolande Lavoie (1972), op. cit. 


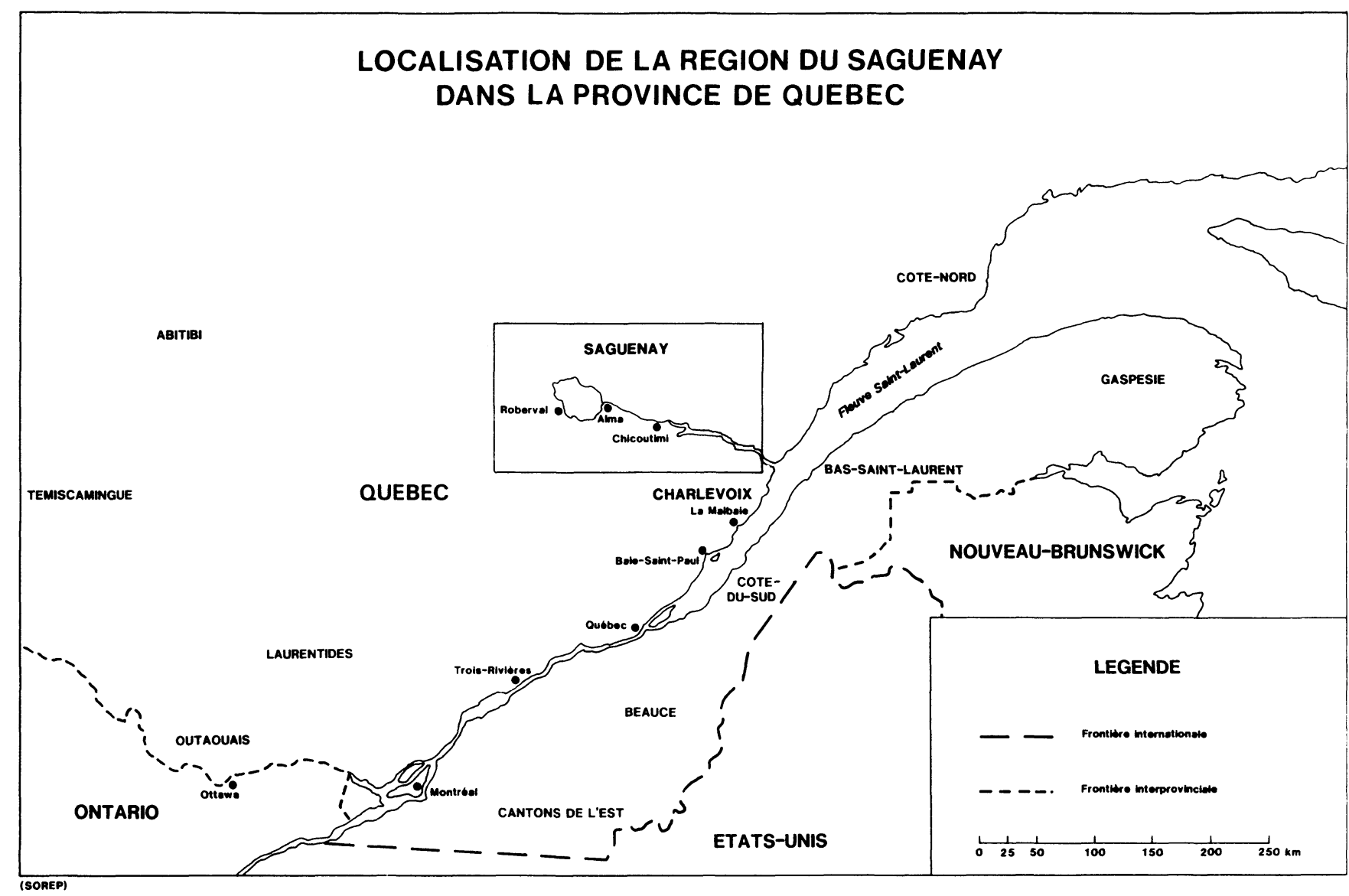


TABLEAU 1

Taux annuels moyens d'accroissement naturel et de migration nette par période décennale Saguenay, 1852-1911

\begin{tabular}{|c|c|c|c|}
\hline Période & $\begin{array}{c}\text { Accroissement } \\
\text { naturel } \\
(\%)\end{array}$ & $\begin{array}{c}\text { Migration } \\
\text { nette } \\
(\%)\end{array}$ & $\begin{array}{c}\text { Accroissement } \\
\text { total } \\
(\%)\end{array}$ \\
\hline $1852-1861$ & 3,6 & $+2,6$ & 6,2 \\
$1862-1871$ & 3,4 & $+1,9$ & 5,3 \\
$1872-1881$ & 3,3 & $-0,3$ & 3,0 \\
$1882-1891$ & 3,3 & $-1,5$ & 1,8 \\
$1892-1901$ & 3,0 & $-0,2$ & 2,8 \\
$1902-1911$ & 3,3 & $-0,3$ & 3,0 \\
\hline
\end{tabular}

Source: Christian Pouyez, Yolande Lavoie et all., Les Saguenayens. Introduction à l' histoire des populations du Saguenay, 16e-20e siècles (Québec, Presses de l'Université du Québec, 1983), 244-245.

annuels d'accroissement naturel se maintiennent à des niveaux élevés et relativement stables qui ne tombent jamais sous le seuil de $3 \%$. Une baisse prévisible suit la période de la première immigration, la structure par âge de la population connaissant un certain vieillissement après l'arrivée des premiers colons ${ }^{25}$.

L'analyse plus fine des mouvements migratoires témoigne d'une différenciation géographique évidente (graphique 1). Ainsi, les taux de migration nette deviennent négatifs beaucoup plus tôt dans le HautSaguenay qu'au Lac-Saint-Jean (voir carte 2 pour la localisation des sous-régions), soit dès le milieu des années 1860 environ. La situation dans le Haut-Saguenay s'améliore toutefois un peu après 1895 , ce qui parait lié au début d'industrialisation que connaît alors cette sous-région. Colonisée plus tardivement et avantagée par l'abondance et la qualité des terres, c'est plus tard que la sous-région du Lac-Saint-Jean connaît des taux de migration nette négatifs.

Les données précédentes sur les soldes migratoires et les taux de migration nette ne permettent cependant pas de connaître l'ampleur respective des mouvements d'immigration et d'émigration. C'est cette limite, importante quand il s'agit de saisir la dynamique démographique, que nous cherchons maintenant à dépasser en mesurant les deux mouvements de façon séparée.

25 Voir Christian Pouyez, «Les composantes de la croissance», Pouyez, Lavoie et al. (1983), op. cit., 278. 


\section{GRAPHIQUE 1}

Taux de migration nette, Haut-Saguenay et Lac St-Jean, 1860-1911.

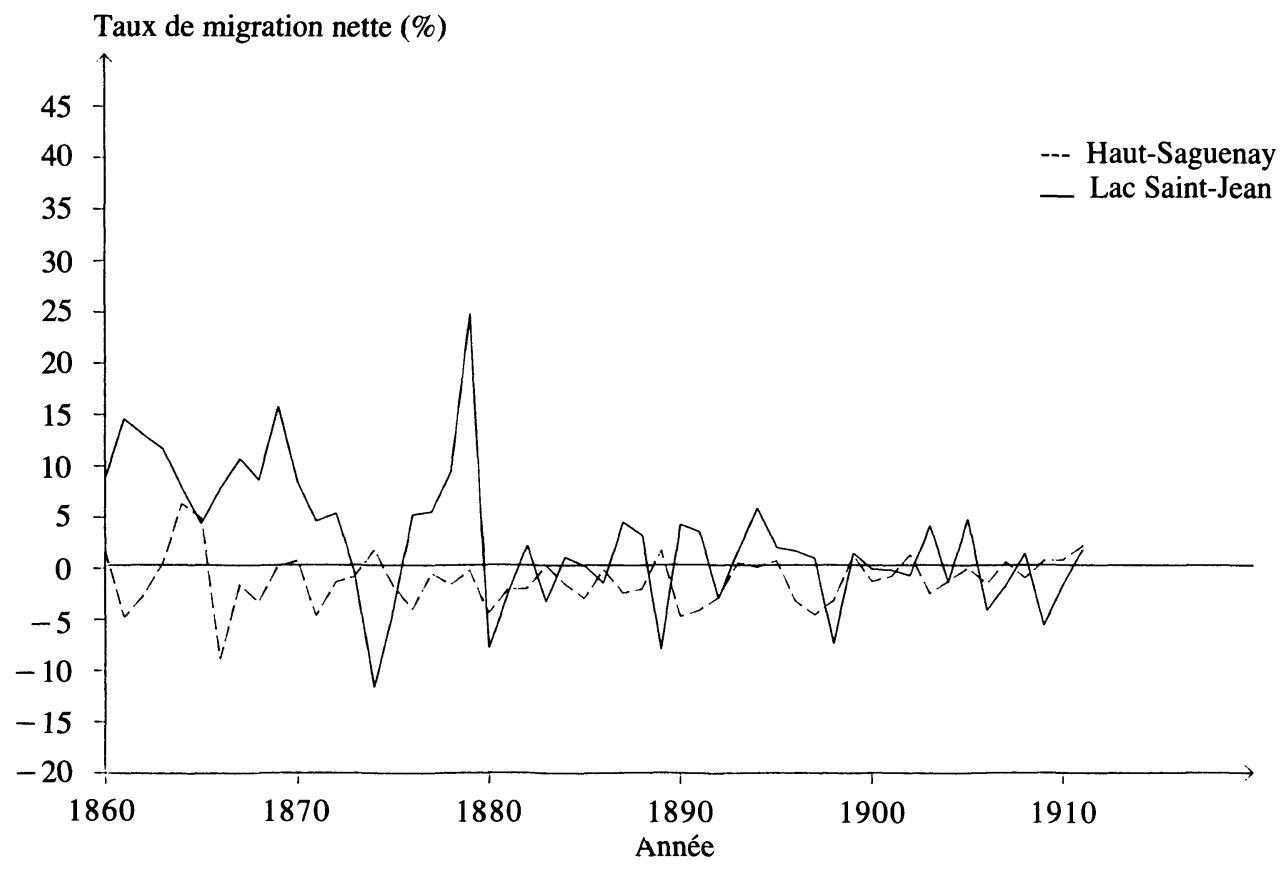

Source: Christian Pouyez, Yolande Lavoie et all., Les Saguenayens. Introduction à l' histoire des populations du Saguenay, 16e-20e siècles (Québec, Presses de l'Université du Québec, 
그

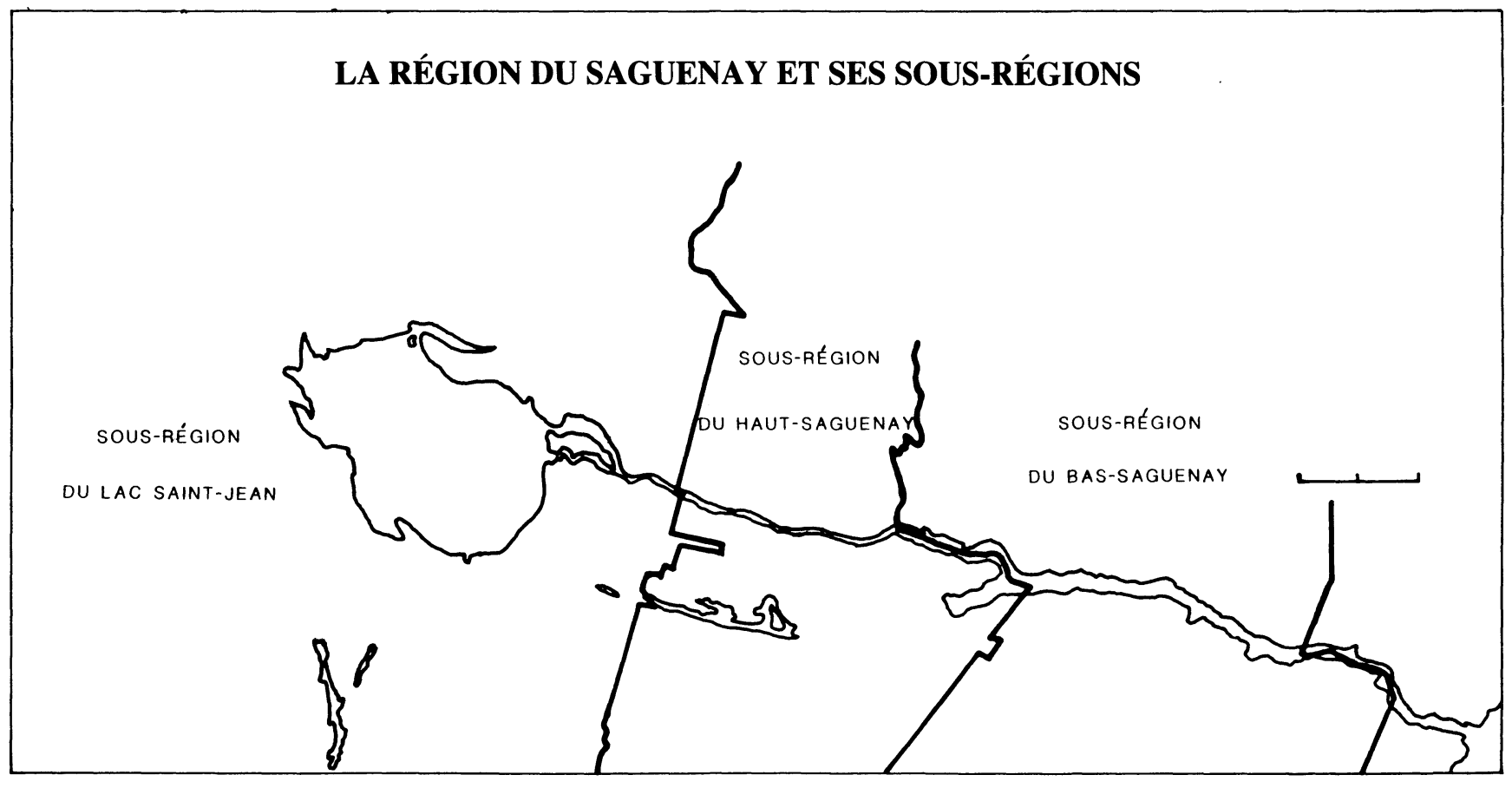

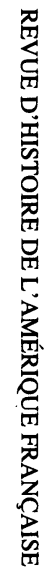




\section{3 - L'AMPLEUR DES MOUVEMENTS MIGRATOIRES}

\section{A - L'utilisation d' une source originale}

Il n'est pas facile de mesurer ni de caractériser les mouvements migratoires, encore moins quand ils concernent les populations du passé. En effet, hormis quelques exceptions ${ }^{26}$, aucune réglementation ne régit l'enregistrement des déplacements des personnes. Les seules façons d'en rendre compte, quand on ne dispose pas de recensements ou de fichiers contemporains adéquats, consistent donc, soit à établir des bilans migratoires nets qui reposent sur des données d'état civil encadrées par deux recensements ${ }^{27}$, soit à comparer des listes nominatives recueillies à divers moments pour y suivre les individus sédentaires et identifier ceux qui migrent ${ }^{28}$. Si la dernière méthode conduit à des résultats plus riches, elle n'en pose pas moins un certain nombre de problèmes liés à la difficulté des jumelages (variations nominatives fréquentes, informations insuffisantes...) et à la longueur de la période qui sépare les deux listes ${ }^{29}$.

Nous nous proposons plutôt d'utiliser ici le fichier des familles reconstituées à partir des registres paroissiaux du Saguenay pour rendre compte des migrations ayant affecté cette région dans le passé. Pour résumer brièvement le principe de cette méthode qui a déjà été décrite ailleurs ${ }^{30}$, disons qu'elle repose sur une utilisation des informations contenues dans les actes de baptême, mariage et sépulture pour saisir les itinéraires migratoires. Ces informations sont utilisées comme autant de points de repères dans les biographies individuelles et familiales. Le concept d'immigration, qui nous intéresse plus particulièrement ici, a été défini en fonction du lieu de naissance, toute personne qui n'est pas née au Saguenay étant qualifiée d'immigrante. L'existence d'un acte de baptême et la déclaration de résidence des parents à cette occasion constituent les éléments essentiels de cette attribution, laquelle a été effectuée pour les 20106 familles reconstituées de la période 18381911.

\footnotetext{
26 Par exemple les registres de population belges ou scandinaves. Voir l'étude de Antti Rosenberg, «Mobility of Population in the Finnish County of Uusimaa (Nyland) 1821-1880», Scandinavian Economic History Review, 14,1-2 (1966): 39-59.

27 C'est ce qui a été fait dans le cas des résultats présentés plus haut.

28 Pour le Saguenay, voir Christian Pouyez, Raymond Roy et Gérard Bouchard, «La mobilité géographique en milieu rural. Le Saguenay, 1852-1861», Histoire sociale/Social History, 14,27 (1981): 123-155.

${ }_{29}$ Voir Donald H. Parkerson, «How Mobile Were Nineteenth-Century Americans?», Historical Methods, 15,3 (1982): 99-109 et Christian Pouyez, Raymond Roy et François Martin, «The Linkage of Census Name Data: Problems and Procedures», Journal of Interdisciplinary History, 14,1 (1983): 129-152.

René Jetté et Danielle Gauvreau, «Des fiches de familles à la mesure des migrations: une méthode élaborée à partir des données du Saguenay au XIXe siècle», Cahiers québécois de démographie, 16,1 (1987): 37-65.
} 


\section{B - Immigration et émigration au Saguenay avant 1911}

La méthode précédente a permis d'identifier 28656 personnes ayant immigré au Saguenay depuis son ouverture à la colonisation vers 1838 jusqu'en 1911. Ce nombre doit généralement être considéré comme un minimum, puisque certains immigrants, le plus souvent des hommes célibataires ayant séjourné temporairement dans la région, n'ont probablement pas laissé de traces dans les registres ${ }^{31}$. Ces 28656 personnes ont été réparties par période d'arrivée (tableau 2), celle-ci étant définie pour les adultes comme la date de leur première mention de couple dans les registres saguenayens, et pour les enfants comme la date de la première mention de leurs parents ${ }^{32}$ (nous reviendrons plus loin sur cette distinction adultes/enfants). Par différence avec les soldes migratoires déjà connus, les nombres d'émigrants ont été estimés indirectement à chaque période. Des taux ont également été calculés.

Les niveaux d'immigration sont évidemment les plus élevés au début de la période, s'atténuant par la suite pour atteindre leur seuil le

TABLEAU 2

Migration nette, immigration et émigration suivant la période Saguenay, 1838-1911

\begin{tabular}{|c|c|c|c|c|c|c|}
\hline Période & $\begin{array}{c}\text { Population } \\
\text { moyenne }\end{array}$ & $\begin{array}{c}\text { Solde } \\
\text { migratoire }\end{array}$ & $\begin{array}{c}\text { Nombre } \\
\text { d'immigrants }\end{array}$ & $\begin{array}{c}\text { Nombre } \\
\text { d'émigrants }\end{array}$ & $\begin{array}{c}\text { Taux annuel } \\
\text { d'immigration } \\
(\%)\end{array}$ & $\begin{array}{c}\text { Taux annuel } \\
\text { d'émigration } \\
(\%)\end{array}$ \\
\hline Avant $1852^{1}$ & - & - & 4285 & - & - & - \\
$1852-1861$ & 7679 & +2010 & 2957 & 947 & 3,9 & 1,2 \\
$1862-1871$ & 13755 & +2591 & 3761 & 1170 & 2,7 & 0,9 \\
$1872-1881$ & 20405 & -699 & 3530 & 4229 & 1,7 & 2,1 \\
$1882-1891$ & 25781 & -3946 & 3095 & 7041 & 1,2 & 2,7 \\
$1892-1901$ & 32682 & -670 & 4569 & 5239 & 1,4 & 1,6 \\
$1902-1911$ & 43792 & -1298 & 6459 & 7757 & 1,5 & 1,8 \\
\hline
\end{tabular}

${ }^{1}$ Le premier recensement ayant été effectué en 1852 , le nombre d'émigrants ne peut être calculé par différence avant la période 1852-1861.

Source: SOREP, familles reconstituées du Saguenay, 1838-1911. Et Pouyez, op. cit., 250.

31 Voir Danielle Gauvreau, René Jetté et Mario Bourque, «Migration in the Saguenay Region: Evidence from Reconstituted Families, 1838-1911», Historical Methods, 20,4 (1987): 143-154. Des résultats de cet article, dont l'objectif était d'abord méthodologique, sont repris ici sous une forme plus synthétique.

32 Une étude menée pour déterminer le moment d'immigration suggère qu'une telle assimilation ne soit pas très éloignée de la réalité (Jetté et Gauvreau (1987), op. cit.). 
plus bas entre 1882 et 1892 , avant de remonter un peu au cours des dernières décennies. De façon générale, l'émigration présente un profil inverse, puisqu'elle augmente d'abord (sauf entre 1862 et 1871) avant de décroître au cours des deux dernières décennies. Tout se passe comme si les mêmes facteurs qui agissaient pour attirer les personnes immigrantes au Saguenay agissaient également pour retenir celles susceptibles de partir. Et, en effet, le début d'industrialisation qui marque la région à la fin du $19 \mathrm{e}$ siècle, peu de temps après la réalisation du chemin de $\mathrm{fer}^{33}$, paraît lié à une certaine reprise du mouvement d'immigration ainsi qu'à un ralentissement de l'émigration. La région se trouve alors rattachée au reste de la province et elle commence à offrir des débouchés industriels dont les immigrants et les émigrants potentiels sont probablement les premiers à profiter: ce sont principalement les usines de pâte à papier qui, s'appuyant sur les ressources hydro-électriques de la région, s'ouvrent à Chicoutimi en 1898 puis à Jonquière, à Val-Jalbert et à Péribonka en 1901. Au contraire, la période précédente est probablement plus exigeante pour les colons dont la vie est toujours aussi difficile, dans un contexte général où l'émigration vers les États-Unis et certaines villes québécoises est importante.

Ces chiffres suggèrent un brassage important de la population: d'une part, celle-ci est alimentée régulièrement par un courant d'immigration qui se prolonge nettement au-delà des premières décennies de colonisation; d'autre part, et parallèlement au mouvement précédent, le Saguenay connaît l'exode d'une fraction importante de ses effectifs, laquelle va jusqu'à $27 \%$ entre 1882 et 1891 et atteint encore $15 \%$ à la fin de la période. Replacés dans le contexte québécois, ces résultats montrent bien comment les migrations de colonisation ne constituent qu'une stratégie parmi d'autres, un essai ou un espoir auquel on n'hésite pas à mettre fin si l'expérience suggère d'autres voies plus prometteuses.

\section{4 - LA COMPOSITION DU MOUVEMENT D'IMMIGRATION}

\section{A - Répartition selon le sexe et statut au moment de l'immigration}

Les hommes et les femmes ayant immigré au Saguenay ont été répartis suivant leur statut familial au moment de l'immigration (tableau 3). Faute d'informations plus complètes, les adultes ont été identifiés comme des personnes dont les parents n'ont pas immigré au Saguenay (ces personnes étaient déjà mariées ou se sont mariées par la suite au Saguenay), les enfants y étant venus avec au moins un parent. Malgré

\footnotetext{
33 Complété en 1888 au Lac-Saint-Jean (Chambord) et en 1893 à Chicoutimi.
} 
TABLEAU 3

Immigrants selon le sexe et le statut au moment de l'immigration Saguenay, 1838-1911

\begin{tabular}{|l|r|r|r|r|r|r|}
\hline \multirow{2}{*}{$\begin{array}{c}\text { Statut au moment } \\
\text { de l'immigration }\end{array}$} & \multicolumn{2}{|c|}{ Femmes } & \multicolumn{2}{c|}{ Hommes } & \multicolumn{2}{c|}{ Sexes réunis } \\
\cline { 2 - 8 } & \multicolumn{1}{c|}{$\mathrm{Nb}$} & \multicolumn{1}{c|}{$\%$} & $\mathrm{Nb}$ & $\%$ & $\mathrm{Nb}$ & $\%$ \\
\hline Enfant & 8168 & 60,5 & 9582 & 63,2 & 17750 & 61,9 \\
Adulte déjà marié & 4816 & 35,7 & 4568 & 30,1 & 9384 & 32,8 \\
Adulte célibataire & 509 & 3,8 & 1013 & 6,7 & 1522 & 5,3 \\
\hline \multicolumn{1}{|c|}{ Ensemble } & 13493 & $100 \%$ & 15163 & $100 \%$ & 28656 & $100 \%$ \\
\hline
\end{tabular}

Source: SOREP, familles reconstituées du Saguenay, 1838-1911.

quelques limites inévitables ${ }^{34}$, cette définition permet généralement de distinguer les personnes autonomes qui sont arrivées seules ou en couples au Saguenay, de celles qui y sont arrivées étant enfants au sein d'une famille.

Dans l'ensemble, le nombre d'immigrants masculins dépasse celui des femmes (le rapport entre les deux étant de 1,12) et la répartition des deux groupes présente des différences statistiquement significatives. La surmasculinité vaut pour toutes les périodes; elle est le fait de deux catégories, les adultes célibataires et les enfants. Le premier cas n'étonne guère, puisque probablement peu de femmes seules émigrent alors de façon autonome. La même situation parmi les enfants suggère quant à elle une certaine sélection de l'immigration en faveur des familles ayant davantage de garçons, sélection qui s'expliquerait par la nature du travail propre à une société de colonisation de type agro-forestier ${ }^{35}$. Cette sélection ne peut cependant s'exercer que sur les familles ayant déjà des enfants suffisamment âgés. Seule la catégorie d'adultes déjà mariés compte un peu plus de femmes que d'hommes: cette différence est légère et pourrait être due à un léger surplus de veuves ayant accompagné des enfants, ou encore à la plus grande propension des hommes à se remarier, ces hommes pouvant aller chercher leur conjointe dans leur milieu d'origine.

\footnotetext{
34 En particulier dans le cas d'enfants orphelins qui auraient immigré avec une soeur ou un frère plus âgé.

35 Voir aussi Gérard Bouchard et Jeannette Larouche, «Dynamique des populations locales: française, 41,3 (hiver 1988): 363-388. L'hypothèse d'une telle sélection est également énoncée dans E. A. Hammel, Sheila R. Johansson et Caren A. Ginsberg, «The Value of Children During Industrialization: Sex Ratios in Childhood in Nineteenth-Century America», Journal of Family History, 8,4 (1983): 346-366.
} 
L'examen des statuts au moment de l'immigration ne laisse aucun doute sur le caractère largement familial de l'immigration, puisque $62 \%$ de tous les immigrants sont des enfants. Même si les cas qui nous ont échappé se concentrent surtout dans la catégorie des adultes célibataires, il est évident qu'on ne peut attribuer à cette dernière qu'une faible fraction des entrées au Saguenay. La répartition enfants/adultes déjà mariés suggère par ailleurs que les couples arrivaient en moyenne avec au moins trois enfants déjà nés (17 750 enfants répartis dans environ 5000 familles, en tenant compte des veufs et des veuves). Comme certains couples immigraient immédiatement après leur mariage, ce nombre est encore supérieur pour ceux formés depuis plus longtemps.

\section{$B$ - Statut migratoire des conjoints et lieu d'établissement}

L'importance de l'apport migratoire pour la région du Saguenay ressort d'une autre façon quand on l'examine du point de vue des conjoints, c'est-à-dire du point de vue de l'ensemble des personnes qui apparaissent au moins une fois à la tête d'un couple. On compte au total 16579 conjoints masculins et 17567 conjoints féminins (tableau 4). La différence entre le nombre de femmes et d'hommes tient essentiellement au fait que ces derniers se remarient davantage que les femmes ${ }^{36}$; ils sont donc moins nombreux pour un nombre semblable d'unions.

\section{TABLEAU 4}

Statut migratoire et lieu du mariage des conjoints selon le sexe Saguenay, 1838-1911

\begin{tabular}{|l|c|c|c|c|c|c|}
\hline \multirow{2}{*}{$\begin{array}{c}\text { Statut migratoire } \\
\text { et lieu du mariage }\end{array}$} & \multicolumn{2}{|c|}{ Femmes } & \multicolumn{2}{c|}{ Hommes } & \multicolumn{2}{c|}{ Sexes réunis } \\
\cline { 2 - 8 } & $\mathrm{Nb}$ & $\%$ & $\mathrm{Nb}$ & $\%$ & $\mathrm{Nb}$ & $\%$ \\
\hline Immigrant & & & & & & \\
- marié à l'extérieur & 4816 & 27,4 & 4568 & 27,6 & 9384 & 27,5 \\
- marié au Saguenay & 4126 & 23,5 & 4831 & 29,1 & 8957 & 26,2 \\
Natif & 8625 & 49,1 & 7180 & 43,3 & 15805 & 46,3 \\
(marié au Saguenay) & & & & & & \\
\hline Ensemble & 17567 & $100 \%$ & 16579 & $100 \%$ & 34146 & $100 \%$ \\
\hline
\end{tabular}

Source: SOREP, familles reconstituées du Saguenay, 1838-1911.

${ }^{36}$ Comme l'ont montré plusieurs études de démographie historique. Voir Jacques Dupâquier, dir., Mariage et remariage dans les populations du passé (London/Toronto, Academic Press, 1981). 
Un peu plus de la moitié des conjoints observés avant 1911 ne sont pas nés au Saguenay, soit $57 \%$ du côté masculin et $51 \%$ du côté féminin. Au total, près du quart des conjoints se sont mariés à l'extérieur du Saguenay. Ces proportions évoluent avec le temps (graphique 2): importantes au début, elles diminuent évidemment par la suite, à mesure que les personnes nées au Saguenay atteignent l'âge de se marier. Elles demeurent cependant non négligeables à la fin de la période, et se stabilisent même alors avec la reprise du mouvement d'immigration. Ainsi, durant les trois dernières décennies observées, un conjoint sur cinq $(21 \%)$, appartenant à un couple qui apparaît pour la première fois dans les registres, est un immigrant arrivé déjà marié. Une telle situation, qui est loin d'être marginale, témoigne de l'importance et de la visibilité certaines de l'immigration saguenayenne jusqu'en 1911.

Nous attardant maintenant aux seuls conjoints mariés au Saguenay, il est étonnant de constater que le rapport de masculinité s'inverse complètement et de façon significative lorsqu'on passe des natifs aux immigrants (respectivement 0,83 et 1,17 ). Visiblement, les femmes natives du Saguenay, plus souvent que les natifs, ont épousé des personnes immigrantes et le déficit observé pour les hommes constitue l'indice d'une émigration individuelle touchant davantage les célibataires masculins. D'autres données renforcent cette hypothèse ${ }^{37}$ qui devra être approfondie dans des travaux ultérieurs.

Comme le suggèrent la marche du peuplement ${ }^{38}$ et les taux de migration nette par sous-région (graphique 1), les nouveaux couples s'installent de moins en moins avec le temps dans le Haut-Saguenay (graphique 3), alors qu'un peu plus du tiers seulement des couples s'y établissent au cours des deux dernières décennies ${ }^{39}$. On sait que c'est surtout le Lac-Saint-Jean qui reçoit les autres couples. Néanmoins, la reprise qui s'amorce avec l'industrialisation est visible à partir de ces résultats. Cette situation fait également voir une différenciation suivant le statut migratoire des conjoints. Voici comment les couples ont été répartis en quatre catégories ${ }^{40}$ :

\footnotetext{
37 Voir Gauvreau, Jetté et Bourque (1987), op. cit.

38 Voir Bouchard (1983), op. cit., 139-164.

39 Le lieu d'établissement a été défini comme le premier lieu de résidence du couple qui apparaît dans leur fiche, hormis le lieu de résidence respectif des conjoints à leur mariage. Il s'agit le plus souvent de la première ou de la seconde mention de résidence possible du couple. Les fiches contenant plus de $50 \%$ de mentions avec résidence indéterminée ont été exclues de cette analyse (près de $10 \%$ de l'ensemble des fiches), ceci afin d'assurer les meilleures conditions de comparaison.

${ }_{40}$ Ces catégories se répartissent évidemment d'une manière différente dans le temps, les couples des groupes 1 et 2 se concentrant davantage au début de la période et ceux du groupe 4 à la fin. Nous tiendrons compte de cet élément dans l'analyse.
} 


\section{GRAPHIQUE 2}

Proportion de conjoints immigrants selon la période

Saguenay, 1838-1911.

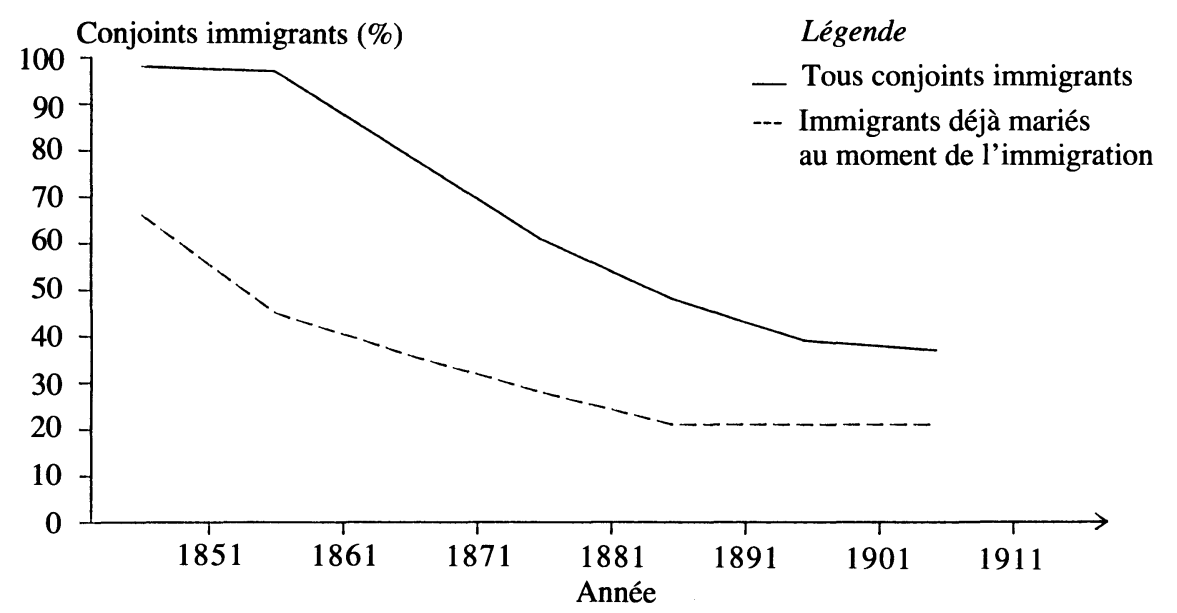

Source: SOREP, familles reconstituées du Saguenay, 1838-1911. 


\section{GRAPHIQUE 3}

Proportion de couples établis au Haut-Saguenay suivant le statut migratoire et la période, Saguenay, 1838-1911.

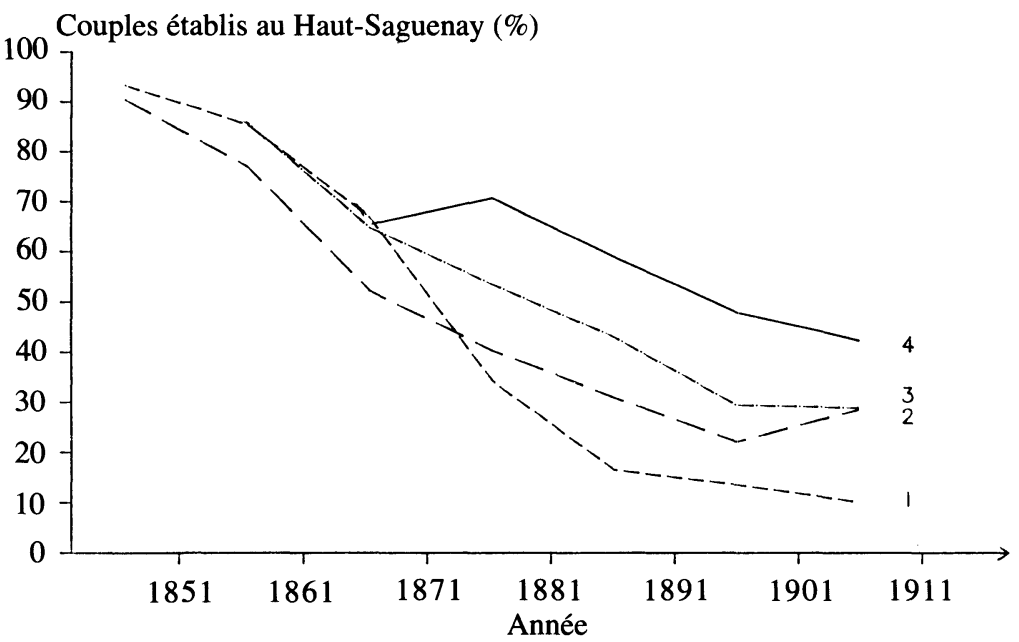

Source: SOREP, familles reconstituées du Saguenay, 1838-1911.

Légende: voir présentation des groupes respectifs dans le texte. 
1 - Deux conjoints immigrants, le couple s'est marié au Saguenay (2 379 couples, soit $16 \%$ de l'ensemble).

2 - Deux conjoints immigrants, le couple est déjà marié à son arrivée au Saguenay (4 128 couples, soit $28 \%$ de l'ensemble).

3 - Un conjoint immigrant, l'autre né au Saguenay (3 273 couples, soit $23 \%$ de l'ensemble).

4 - Deux conjoints nés au Saguenay (4 802 couples, soit 33\% de l'ensemble).

Il ressort tout d'abord que l'on s'établit en moins grand nombre dans le Haut-Saguenay lorsqu'on est immigrant. Cette relation est très nette à partir de 1870 environ, alors que les couples d'immigrants mariés au Saguenay sont proportionnellement les moins nombreux à s'établir au Haut-Saguenay, à l'opposé des couples où les deux conjoints sont natifs. Visiblement, la sous-région du Haut-Saguenay atteint assez rapidement une limite dans sa capacité d'intégration des nouveaux arrivants, agriculteurs pour la plupart, étant donné la rareté et le coût croissant des terres. Alors même que de nombreuses familles du Haut-Saguenay émigrent vers le Lac pour s'établir avec leurs enfants ${ }^{41}$, il est facile d'imaginer que les possiblités d'établissement pour les personnes nouvellement arrivées ne sont pas nombreuses. Cette situation connaît toutefois un début de renversement au cours de la dernière décennie, alors que la proportion de couples impliquant au moins un conjoint immigrant qui s'établit dans le Haut-Saguenay se stabilise ou accuse une légère augmentation. La même chose n'apparaît pas chez les natifs (groupe 4) qui, mieux enracinés dans la société rurale saguenayenne, ne sont probablement pas les premiers à s'engager dans le mouvement d'industrialisation.

\section{C - Origines géographiques du courant migratoire}

Diverses sources ont déjà démontré l'existence d'un réseau migratoire privilégié entre le Saguenay et la région de Charlevoix, située à l'est de Québec sur la rive nord du Saint-Laurent ${ }^{42}$. Ainsi, la plupart des colons qui sont venus au Saguenay au début provenaient de cette région. Souvent, ils quittaient une situation économique difficile où les terres agricoles devenaient de plus en plus rares et coûteuses et où l'ouverture de nouvelles paroisses sur le plateau ne satisfaisait plus aux besoins. Mais le mouvement d'immigration s'alimentait aussi à même d'autres régions de l'est du Québec ainsi que, dans une moindre mesure probablement, à l'ouest de la province.

Les sources saguenayennes d'information sur la population ne permettent de tracer qu'un portrait incomplet de l'origine géographique 
des personnes immigrantes. Seul le recensement de 1852 donne des lieux de naissance suffisamment détaillés. Les lieux de résidence déclarés dans les registres à l'occasion du mariage ne sont utiles que pour le groupe des conjoints (ou leurs parents) ne résidant pas au Saguenay au moment de leur mariage. Pour compléter ce tableau, il a fallu rechercher plus systématiquement les régions d'origine des conjoints immigrants du Saguenay à partir de répertoires régionaux de mariages. Cette recherche s'est faite dans les répertoires de Charlevoix d'abord, puis du reste de l'est du Québec, c'est-à-dire la partie du Québec comprenant la ville même de Québec et tout ce qui se trouve à l'est. Le lieu du mariage du couple, ou celui de ses parents si le couple s'est marié au Saguenay, a été retenu comme lieu d'origine, après vérification que cette différence n'introduisait pas de biais important. Les autres origines inconnues sont vraisemblablement situées surtout dans l'ouest du Québec et exceptionnellement aux États-Unis ou au Canada. Le travail, réalisé sur deux échantillons de 300 conjoints masculins et 300 conjoints féminins, a également servi à identifier les personnes du même groupe familial (parents - enfants - frères/soeurs) ayant immigré au Saguenay en provenance des mêmes régions, et donc à évaluer le caractère plus ou moins familial de l'immigration, en fonction particulièrement du lieu d'origine ${ }^{43}$.

Les résultats confirment l'importance de la région de Charlevoix comme lieu d'origine principal des personnes immigrantes, particulièrement au cours des premières décennies (graphique 4). Ils font cependant ressortir aussi la diversification précoce du bassin de provenance au profit des autres régions de l'est du Québec, puis surtout des autres lieux d'origine. Les hommes et les femmes présentent à cet égard un profit très semblable, c'est pourquoi ils n'ont pas été distingués.

Une nette différence apparaît toutefois dans la situation des conjoints mariés à l'extérieur du Saguenay, et donc arrivés déjà mariés, et celle des conjoints mariés au Saguenay. Ces derniers proviennent en effet plus souvent de la région de Charlevoix, ce qui paraît lié à au moins deux facteurs: d'une part, l'immigration en provenance de Charlevoix s'étant faite surtout au début de la période, les enfants immigrants dont les parents se sont mariés dans Charlevoix sont plus nombreux à s'être mariés avant 1911, date qui marque la fin de la période étudiée; d'autre part, un taux d'enracinement au Saguenay plus élevé pour les personnes de Charlevoix paraît également en cause ici ${ }^{44}$.

\footnotetext{
43 Une présentation de ces deux échantillons apparaît dans Manon Declos, Danielle Gauvreau et Mario Bourque, Présentation de deux échantillons et du guide de relevé utilisés pour l'étude de l'immigration au Saguenay, 1838-1911 (Chicoutimi, Université du Québec à Chicoutimi, Document de SOREP no II-C-126, 1986).

44 Raymond Roy, Peuplement primitif et courants migratoires. Communication présentée au Symposium international SOREP, Chicoutimi, septembre 1987.
} 


\section{GRAPHIQUE 4}

Origine géographique des immigrants suivant la période,

Saguenay, 1838-1911.

Immigrants déjà mariés au moment de l'immigration

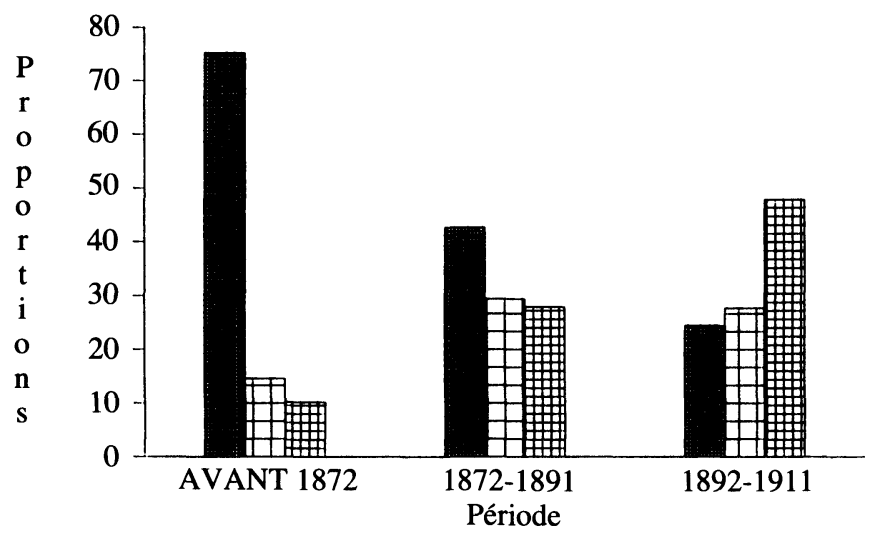

Immigrants mariés au Saguenay

Légende

Charlevoix

Autre -

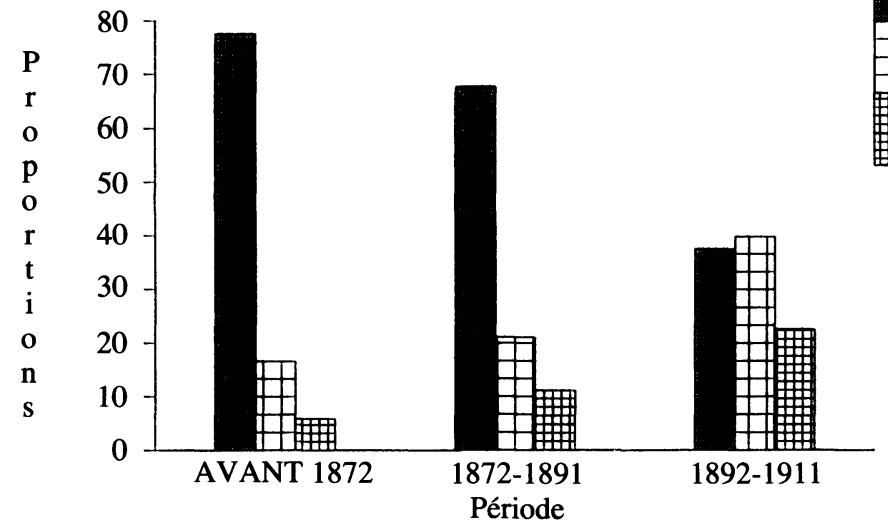

Est du Québec

Autre -

Inconnu

Source: SOREP, étude basée sur deux échantillons de conjoints immigrants, Saguenay, 1838-1911. 
Hormis Charlevoix, les régions de l'est du Québec qui ont le plus nourri le courant d'immigration vers le Saguenay sont la région de Québec (ville et environs) et la Côte-du-Sud, suivies de la Côte-deBeaupré, du Bas-Saint-Laurent, puis de la Côte-Nord et de la Beauce. $\mathrm{Au}$ sein de Charlevoix, toutes les paroisses sont représentées, ce qui indique bien que le phénomène d'émigration n'est pas le fait de quelques contributions ponctuelles (tableau 5). La comparaison avec la répartition de la population de Charlevoix au recensement de $1861^{45}$ met cependant en évidence la surreprésentation des plus grosses paroisses comme Baie-Saint-Paul et la Malbaie, alors qu'à l'opposé les paroisses plus petites ouvertes plus récemment (Saint-Hilarion, Saint-Irénée, Saint-Fidèle, Saint-Siméon) ou plus isolées (Petite-Rivière, l'Ile-auxCoudres) sont un peu sous-représentées.

\section{TABLEAU 5}

Origine dans Charlevoix des immigrants au Saguenay (1838-1911) et répartition de la population de Charlevoix en 1861

\begin{tabular}{|l|r|r|r|r|r|r|}
\hline \multirow{2}{*}{ Lieu d'origine } & \multicolumn{2}{c|}{$\begin{array}{c}\text { Immigrants } \\
\text { arrivés déjà } \\
\text { mariés }\end{array}$} & \multicolumn{2}{c|}{$\begin{array}{c}\text { Immigrants } \\
\text { mariés au } \\
\text { Saguenay }\end{array}$} & \multicolumn{2}{c|}{$\begin{array}{c}\text { Population de } \\
\text { Charlevoix en } \\
1861\end{array}$} \\
\cline { 2 - 7 } & \multicolumn{1}{|c|}{$\mathrm{Nb}$} & \multicolumn{1}{c|}{$\%$} & $\mathrm{Nb}$ & \multicolumn{1}{c|}{$\%$} & $\mathrm{Nb}$ & $\%$ \\
\hline Petite-Rivière-Saint-François & 4 & 2,7 & 6 & 3,5 & 728 & 4,8 \\
Baie-Saint-Paul & 44 & 30,1 & 49 & 28,8 & 3664 & 24,1 \\
Les Éboulements & 18 & 12,3 & 24 & 14,1 & 2235 & 14,7 \\
Île aux Coudres & 5 & 3,4 & 7 & 4,1 & 700 & 4,6 \\
Saint-Urbain & 10 & 6,8 & 15 & 8,8 & 761 & 5,0 \\
Saint-Hilarion & - & - & - & - & 540 & 3,5 \\
Saint-Irénée & 3 & 2,0 & 1 & 0,6 & 998 & 6,5 \\
La Malbaie & 41 & 28,1 & 46 & 27,1 & 2766 & 18,2 \\
Sainte-Agnès & 17 & 11,6 & 22 & 12,9 & 1722 & 11,3 \\
Saint-Fidèle & 3 & 2,0 & - & - & 836 & 5,5 \\
Saint-Siméon & - & - & - & - & 273 & 1,8 \\
\hline \multicolumn{1}{|c|}{ Ensemble } & 146 & $100 \%$ & 170 & $100 \%$ & 15223 & $100 \%$ \\
\hline
\end{tabular}

Source: SOREP, étude basée sur deux échantillons de conjoints immigrants, Saguenay, 18381911 et recensement du Bas Canada en 1861.

\footnotetext{
45 Ce recensement a été retenu comme point de comparaison parce que l'essentiel de l'immigration en provenance de Charlevoix s'est faite au cours des premières décennies de colonisation. De toute façon, la répartition au sein même de la population de Charlevoix évolue peu d'une décennie à l'autre: à la fín de la période, elle témoigne seulement de l'importance accrue des paroisses ouvertes plus récemment. Du reste, le phénomène rapporté ici a aussi été observé pour la décennie 1842-1851 (voir Roy (1987): op. cit.).
} 


\section{D - Le caractère familial de l'immigration}

Ajoutant aux résultats précédents sur le statut familial des conjoints au moment de l'immigration, la recherche plus approfondie menée sur deux échantillons d'immigrants démontre à son tour que l'immigration vers le Saguenay n'est pas surtout le fait de personnes seules venues s'installer dans une nouvelle région de colonisation. Cette fois l'accent est mis davantage sur l'apparentement, c'est-à-dire sur la venue de personnes unies par des liens biologiques de premier degré (parents, frères/ soeurs, enfants).

La majorité des personnes immigrantes sont liées de cette manière avec d'autres personnes venues au Saguenay, surtout celles en provenance de Charlevoix (tableau 6). Parmi ces dernières, seulement 6\% n'ont pas de lien de premier degré avec un autre immigrant, cette proportion atteignant $33 \%$ chez les personnes mariées dans d'autres régions. La taille moyenne du noyau familial immigrant est de 6,8 pour le premier groupe et de 3,3 pour le second; cette différence persiste lorsque le calcul exclut les personnes seules. Elle tient en partie au stade plus précoce de la famille au moment de son immigration au Saguenay: en effet, les personnes mariées dans Charlevoix immigrent en moyenne 17,9 ans après le mariage, cette durée étant de 13,4 pour celles mariées à l'extérieur qui ont alors moins d'enfants susceptibles de migrer avec elles ou dans leur sillon. La différence s'explique aussi par un écart en faveur de Charlevoix dans le nombre de frères et soeurs déjà mariés

\section{TABLEAU 6}

Quelques statistiques concernant le caractère familial de l'immigration suivant la catégorie des immigrants Saguenay, 1838-1911

\begin{tabular}{|c|c|c|c|}
\hline \multirow[b]{2}{*}{ Caractère familial de l'immigration } & \multicolumn{2}{|c|}{ Immigrants arrivés déjà mariés } & \multirow[b]{2}{*}{$\begin{array}{c}\text { Immigrants mariés } \\
\text { au Saguenay }\end{array}$} \\
\hline & $\begin{array}{l}\text { Mariés dans } \\
\text { Charlevoix }\end{array}$ & $\begin{array}{c}\text { Mariés à l'extérieur } \\
\text { de Charlevoix }\end{array}$ & \\
\hline Proportion d'immigrants isolés & $6 \%$ & $33 \%$ & $4 \%$ \\
\hline $\begin{array}{l}\text { Taille moyenne du groupe familial } \\
\text { migrant }\end{array}$ & 6,8 & 3,3 & 6,2 \\
\hline $\begin{array}{l}\text { Nombre moyen d'années séparant la } \\
\text { venue du premier et du dernier immigrant } \\
\text { du groupe familial }\end{array}$ & 16,9 & 6,3 & 12,8 \\
\hline Nombre total de cas & 143 & 62 & 237 \\
\hline
\end{tabular}

Source: SOREP, étude basée sur deux échantillons de conjoints immigrants, Saguenay, 18381911. 
ayant immigré au Saguenay. Visiblement, le réseau migratoire familial est plus étendu du côté de Charlevoix, ce qui est cependant aussi facilité par une immigration plus précoce au Saguenay (graphique 4). Il n'y a pas de différence significative du coté des parents: dans l'ensemble, $25 \%$ des immigrants déjà mariés ont également leur père ou leur mère qui immigre dans la région ( $15 \%$ ont les deux), proportion relativement élevée qui témoigne encore du caractère familial de l'immigration, lequel ne se limite pas à la cellule conjugale immédiate.

L'immigration du groupe familial ne s'effectue pas nécessairement à un seul moment ou dans une courte période. En effet, la durée moyenne qui sépare la migration de la première et de la dernière personne du groupe est de 16,9 ans pour les personnes mariées dans Charlevoix et de 6,3 pour les autres, un résultat qui découle évidemment de la différence observée dans la taille des groupes. La variété des situations est certes considérable. Le cas le plus simple concerne des couples déjà mariés arrivés au Saguenay avec des enfants qui s'établissent à leur tour dans la région. D'autres cas témoignent de stratégies plus complexes et de séjours souvent temporaires dans la région. Ainsi, tel couple des Éboulements a un garçon qui se marie et s'établit à Laterrière en 1864. À peu près en même temps, deux de ses soeurs déjà mariées arrivent à Laterrière (Haut-Saguenay), puis vont s'établir à Hébertville (Lac-Saint-Jean); aucune des deux ne persiste toutefois dans la région. Une autre fille connaît au Lac-Saint-Jean un cheminement semblable à la fin des années 1870. Enfin, deux garçons et une fille déjà mariés s'établissent dans la région avec leur famille (au moins jusqu'en 1911): la première vers 1870 au Haut-Saguenay, puis au Lac-Saint-Jean; les deux garçons arrivent vers la fin des années 1870, l'un au Lac-SaintJean et l'autre au Bas-Saguenay puis au Lac-Saint-Jean. À la fin du 19e siècle et jusqu'en 1911 (fin d'observation), trois garçons et une fille sont encore présents dans la région: l'un est toujours à Laterrière tandis que les trois autres sont tous au Lac-Saint-Jean, mais dans des paroisses différentes (Lac-Bouchette, Saint-Bruno et Hébertville). Ce cas ne constitue bien sûr qu'un exemple, mais comme bien d'autres itinéraires, il suggère l'existence de réseaux migratoires familiaux dont les ramifications s'étendent dans le temps et l'espace; il rend également compte du caractère temporaire de plusieurs établissements ${ }^{46}$.

La situation des personnes mariées au Saguenay est un peu différente et traduit une double réalité (tableau 6): d'un côté, celle des célibataires qui arrivent de façon isolée; de l'autre, celle des enfants qui arrivent avec leurs parents et d'autres frères et soeurs déjà mariés ou qui se marient au Saguenay. Les résultats obtenus témoignent de cette situation mixte où domine nettement le second groupe.

\footnotetext{
46 Voir aussi l'étude approfondie de Marc Saint-Hilaire pour la paroisse de Saint-Fulgence, «Origines et destins des familles pionnières d'une paroisse saguenayenne au 19e siècle», Cahiers de géographie du Québec, 32,85 (1988): 5-26.
} 


\section{CONCLUSION}

Sous la poussée d'un mouvement de colonisation, le 19e siècle québécois voit s'ouvrir de nombreuses régions dont le peuplement vient modifier sensiblement la carte du Québec. Façonnant la réalité régionale actuelle et passée, ce mouvement ne constitue toutefois qu'un volet dans le vaste ensemble de mouvements migratoires qui affectent alors la population. Ainsi, nous avons estimé ici que 28000 personnes sont entrées au Saguenay avant 1911, alors que des centaines de milliers d'autres traversaient la frontière pour aller travailler aux États-Unis. Même si les chiffres saguenayens semblent modestes, il s'agit à l'échelle régionale d'un phénomène majeur qui donne le coup d'envoi au développement d'une région qui compte aujourd'hui près de 300000 personnes.

L'analyse précédente démontre bien que les couples et les familles sont les acteurs principaux de ce mouvement. Ce résultat vient s'ajouter à ceux de quelques études récentes, renforçant ainsi l'hypothèse selon laquelle la famille et l'exploitation familiale occupent une place centrale dans le modèle de reproduction démographique et sociale qui s'appuie alors largement sur la mobilité géographique. Comme le suggèrent Darroch, puis Bouchard ${ }^{47}$, la migration vécue dans de telles conditions ne constitue pas un élément déstabilisateur mais plutôt la pièce essentielle d'un processus de reproduction. Ses conséquences pour la société d'origine comme pour la société d'accueil demandent cependant à être approfondies, tout comme les diverses facettes du rôle de la famille dans ce mouvement: élaboration de stratégies migratoires, constitution de réseaux d'information et de soutien au point de départ et à l'arrivée, établissement des enfants...

Au Saguenay, le caractère familial de l'immigration fondatrice n'est pas sans conséquence pour l'homogénéité de la population nouvellement implantée. Cette homogénéité se trouve même accentuée par l'existence d'un couloir privilégié qui s'établit, du moins dans les premières décennies, entre Charlevoix et le Saguenay. L'avance charlevoisienne ainsi prise dans la constitution du bassin génétique saguenayen est encore amplifiée par l'apparentement plus marqué de ce groupe de migrants.

47 Gordon A. Darroch, «Migrants in the Nineteenth Century: Fugitives or Families in Motion?», Journal of Family History, 6,3 (1981): 257-277. Gérard Bouchard, «La dynamique communautaire et l'évolution des sociétés rurales québécoises aux $19 \mathrm{e}$ et $20 \mathrm{e}$ siècles. Construction d'un modèle», Revue d' histoire de l'Amérique française, 40,1 (été 1986): 51-71. 
La question qui surgit dès lors concerne le destin ultérieur des immigrants et immigrantes au Saguenay. Importante du point de vue historique et démographique, mais aussi génétique, cette question commande une analyse plus fine des cheminements migratoires, en particulier de l'émigration, de façon à cerner la dynamique différentielle d'enracinement au Saguenay. Dès 1870, des taux d'émigration élevés viennent compenser l'apport migratoire qui se poursuit au-delà de la période de première colonisation. Comme certaines études sur les migrations le suggèrent, il est probable que les personnes récemment arrivées soient les plus susceptibles de migrer à nouveau ${ }^{48}$, même si nos résultats indiquent également l'existence de départs de natifs. Ainsi s'établirait dans la région un noyau plus stable de familles, autour duquel graviterait une population plus mouvante, largement affectée par l'immigration et l'émigration. Un tel modèle s'accorde avec les hypothèses concernant la prévalence élevée de certaines maladies génétiques au Saguenay et paraît confirmé par les résultats obtenus pour la période antérieure à $1862^{49}$. Il fait actuellement l'objet de recherches plus approfondies.

48 Voir par exemple Sydney Goldstein, «The Extend of Repeated Migration: an Analysis Based on the Danish Population Register», Journal of the American Statistical Association, 59 (1964): 1121-1132.

49 Roy (1987), op. cit. 